\title{
ARTICLE
}

Received 18 Apr 2013 | Accepted 24 Oct 2013 | Published 2 Dec $2013 \quad$ DOl: 10.1038/ncomms3819

\section{Renewable and metal-free carbon nanofibre catalysts for carbon dioxide reduction}

\author{
Bijandra Kumar ${ }^{1}{ }^{\star}$, Mohammad Asadi $^{1, \star}$, Davide Pisasale${ }^{1}$, Suman Sinha-Ray ${ }^{1}$, Brian A. Rosen², \\ Richard Haasch ${ }^{3}$, Jeremiah Abiade ${ }^{1}$, Alexander L. Yarin ${ }^{1,4} \&$ Amin Salehi-Khojin ${ }^{1}$
}

The development of an efficient catalyst system for the electrochemical reduction of carbon dioxide into energy-rich products is a major research topic. Here we report the catalytic ability of polyacrylonitrile-based heteroatomic carbon nanofibres for carbon dioxide reduction into carbon monoxide, via a metal-free, renewable and cost-effective route. The carbon nanofibre catalyst exhibits negligible overpotential $(0.17 \mathrm{~V})$ for carbon dioxide reduction and more than an order of magnitude higher current density compared with the silver catalyst under similar experimental conditions. The carbon dioxide reduction ability of carbon nanofibres is attributed to the reduced carbons rather than to electronegative nitrogen atoms. The superior performance is credited to the nanofibrillar structure and high binding energy of key intermediates to the carbon nanofibre surfaces. The finding may lead to a new generation of metal-free and non-precious catalysts with much greater efficiency than the existing noble metal catalysts.

\footnotetext{
${ }^{1}$ Department of Mechanical and Industrial Engineering, University of Illinois at Chicago, Chicago, Illinois 60607, USA. ${ }^{2}$ Department of Chemical and Biomolecular Engineering, University of Illinois at Urbana-Champaign, Urbana, Illinois 61801, USA. ${ }^{3}$ Materials Research Laboratory, University of Illinois at Urbana-Champaign, Urbana, Illinois 61801, USA. ${ }^{4}$ College of Engineering, Korea University, Seoul 136-713, South Korea. * These authors contributed equally to this work. Correspondence and requests for materials should be addressed to B.K. (email: bijandra@uic.edu) or to A.S.-K. (email: salehikh@uic.edu).
} 
recent report on the future of carbon dioxide $\left(\mathrm{CO}_{2}\right)$ emission ${ }^{1}$ predicts that in the coming few decades (20102060), 496 gigatonnes of $\mathrm{CO}_{2}$ will be produced because of fossil fuel combustion by existing infrastructure. Interestingly, $\mathrm{CO}_{2}$ can be artificially converted into fuel or commodity chemicals $^{2,3}$. The $\mathrm{CO}_{2}$ conversion methodology not only addresses the potential solution for controlling the $\mathrm{CO}_{2}$ concentration level in the environment but also offers an alternative approach for conversion of renewable energy to a chemical fuel or product. Thus, more research efforts are emphasized towards the $\mathrm{CO}_{2}$ conversion by electrochemical reduction ${ }^{4-10}$. So far, various noble metals $\left(\mathrm{Ag}^{4,10}, \mathrm{Au}^{6}, \mathrm{Cu}^{7}, \mathrm{Pt}^{8}\right.$ and so on) and metal complexes ${ }^{9}$ are used as heterogeneous catalysts (as electrodes) for $\mathrm{CO}_{2}$ reduction. However, the rising cost of noble metals is the main hindrance towards their largescale practical applications.

As a substitute, non-metallic homogenous catalysts (that is, in liquid media) have been significantly studied for the $\mathrm{CO}_{2}$ reduction ${ }^{2,11-13}$. Nevertheless, stability, tedious product separation processes and recovery of the catalyst in these systems still remain among the most problematic issues ${ }^{14}$. On the other hand, a relatively new class of heteroatomic, metal-free heterogeneous catalysts (for example, nitrogen-doped carbon nanotube ${ }^{15}$ ) have shown high catalytic performance comparable to noble metal catalysts (for example, platinum) for oxygen reduction reaction (ORR) ${ }^{15-19}$. The insertion of heteroatoms (for example, nitrogen and sulphur $)^{17}$ in the carbon lattice converts the neutral carbon atoms into positively charged atoms (active sites). It has been claimed that the $\mathrm{O}-\mathrm{O}$ bond is effectively weakened because of parallel diatomic adsorption of oxygen molecules on these active sites, which promotes the ORR ${ }^{15-19}$. However, $\mathrm{CO}_{2}$ reductions' mechanistic path is entirely different from the ORR as $\mathrm{CO}_{2}$ reduction occurs via the formation of intermediate $\mathrm{CO}_{2}$ complex species, in particular when ionic liquid is used as the electrolyte ${ }^{4}$. Unexpectedly, there are rarely reports on non-metallic heterogeneous catalysts for $\mathrm{CO}_{2}$ reduction; none have succeeded in demonstrating satisfactory $\mathrm{CO}_{2}$ reduction ability. In addition, $\mathrm{CO}_{2}$ reduction mechanism for carbon nitrogen heteroatomic catalysts has not been addressed ${ }^{20}$.

In this study, we use low-priced and metal-free carbon nanofibres (CNFs) catalyst synthesized by pyrolysis of electrospun nanofibre mats of heteroatomic polyacrylonitrile (PAN) polymer ${ }^{21}$ for $\mathrm{CO}_{2}$ reduction. The heteroatomic configuration of this catalyst is designed to exploit existing nitrogen atoms in the backbone of precursor (PAN). 1-ethyl-3-methylimidazolium tetrafluoroborate $\left(\mathrm{EMIM}-\mathrm{BF}_{4}\right)$ is chosen as the electrolyte because of its high $\mathrm{CO}_{2}$ capture ability ${ }^{22}$ and $\mathrm{CO}_{2}$ reduction overpotential-dropping feature ${ }^{4}$. Our non-metallic catalyst system demonstrates the highest performance for $\mathrm{CO}_{2}$ reduction at low overpotential compared with existing noble metal catalysts to date (for example, $\sim 13$ times higher $\mathrm{CO}_{2}$ reduction current density compared with bulk Ag catalyst).

\section{Results}

CNFs characterization. The CNFs were synthesized by carbonization of electrospun PAN nanofibre mats in argon (Ar) media (see Methods). Macroscopic image of an as-prepared CNFs mat (a defined small piece) is shown in Fig. 1a. The scanning electron microscopic (SEM) image shows the morphological view (at micro scale) of the sample displaying randomly oriented nanofibres in the mat (Fig. 1b). The magnified SEM and atomic force microscopic images (Fig. 1c-e) deliver more detailed information about the dimensions of the individual fibre (the cross-sectional diameter $\sim 500 \mathrm{~nm}$ ). The surface roughness along the axis of an individual fibre was also measured resulting in an average surface corrugation with a height of $\sim 15 \mathrm{~nm}$ (Fig. 1f). The nanocorrugated fractal-like configuration also dramatically increases the effective surface area. High-resolution transmission electron microscopy verified the short-range ordered crystal below few nanometres, which clearly manifests the turbostratic nature of the $\mathrm{CNFs}^{21}$. The diffused ring in the selected area electron diffraction pattern (inset in Fig. 1g) is also consistent with the TEM analysis, confirming the disordered crystal (graphitic) structure of CNFs surface.

In addition to morphological characterizations, the graphitic nature and atomic arrangement was investigated by performing Raman spectroscopy and X-ray photoelectron spectroscopy (XPS). Figure $1 \mathrm{~h}$ represents the typical Raman spectrum of CNFs indicating two identical peaks that is, D band and $G$ band at $\sim 1,365$ and $\sim 1,586 \mathrm{~cm}^{-1}$, respectively. In general, the $G$ band occurs because of an $\mathrm{E}_{2 \mathrm{~g}}$ hexagonal graphitic lattice, whereas the $\mathrm{D}$ band (owing to defects) corresponds to the $\mathrm{A}_{1 \mathrm{~g}}$ mode ${ }^{23}$. In particular, substitution of carbon atoms by other species is accompanied by the introduction of defects into the carbon lattice. The increased $\mathrm{D}$ band to $\mathrm{G}$ band intensity ratio $\left(\mathrm{I}_{\mathrm{D}} / \mathrm{I}_{\mathrm{G}}\right)$, which is nearly one, $(\sim 0.81)$ suggests the presence of defects in the carbon lattice ${ }^{24}$. It is emphasized that nitrogen atoms (as defects) are supposed to be one major component of CNFs. Thus, besides Raman spectroscopy, XPS was also performed to study the atomic configuration of different elements within materials. In the XPS spectrum of CNF, the peaks appearing at binding energy (B.E.) about 400 and $284 \mathrm{eV}$ correspond to the $\mathrm{N} 1 s$ and $\mathrm{C} 1 s$, respectively (Supplementary Fig. S1) ${ }^{25}$. The existence of N1s confirms the presence of nitrogen atoms even if CNFs were pyrolysed at $1,050^{\circ} \mathrm{C}$ in Argon media. These results strongly suggest that presence of nitrogen atoms is only due to the native heteroatomic structure of PAN. Further, C1s peak was fully analysed. The deconvoluted $\mathrm{C} 1 s$ peak consists of mainly two individual peaks that are assigned to $\mathrm{C}-\mathrm{C}, \mathrm{C}=\mathrm{C}$ bond (B.E. $\sim 284.8 \mathrm{eV}$ ) and $\mathrm{C}-\mathrm{N}$ bond (B.E. $\sim 286.01 \mathrm{eV}$ ). An insignificant peak at $287.8 \mathrm{eV}$ was also observed, which indicates the existence of $\mathrm{O}=\mathrm{C}-\mathrm{N}$ type of structures (Fig. 1i).

$\mathrm{CO}_{2}$ reduction performance of the CNFs catalyst. The $\mathrm{CO}_{2}$ reduction efficiency of CNFs catalysts has been examined by sweeping the applied voltage from 0.79 to $-1.14 \mathrm{~V}$ versus the standard hydrogen electrode (SHE) as shown in Fig. 2a. The cyclic voltammetry $(\mathrm{CV})$ curve acquired in the presence of $\mathrm{CO}_{2}$ - saturated EMIM-BF 4 shows that two well-defined peaks appear at $-0.573 \mathrm{~V}$ versus $\mathrm{SHE}$ and $-1.14 \mathrm{~V}$ versus SHE, respectively. This first peak is the $\mathrm{CO}_{2}$ reduction characteristic peak, similar to the results reported (Ag-induced $\mathrm{CO}_{2}$ reduction) previously ${ }^{10,26,27}$. To validate the reaction yields at the first peak, the potential was held constant at $-0.573 \mathrm{~V}$ (versus SHE) and the collected products were analysed by gas chromatography (GC). We observed a Faradaic efficiency of $98 \%$ for carbon monoxide (CO) formation. This means that almost all of the energy (electrons) of the system was used for the ' $\mathrm{CO}_{2}$ conversion to CO' process.

To evaluate the role of the corrugated nanofibrillar structure, additional $\mathrm{CV}$ experiments were performed with carbon films (C film) prepared by PAN under similar pyrolysis conditions. SEM images clearly demonstrate that C film (Supplementary Fig. S2) neither possesses the overall porosity of nanofibre mats nor the fractal-like corrugations characteristic of the individual fibres. The XPS and Raman spectra analyses (Supplementary Figs S3-S6) confirm a similar chemical structure of $\mathrm{C}$ films and CNFs. The first salient feature in the $\mathrm{CV}$ curves is that the onset potential for $\mathrm{CO}_{2}$ reduction (to $\mathrm{CO}$ ) in $\mathrm{CNFs}$ is $\sim 0.23 \mathrm{~V}$ lower than that recorded for the $\mathrm{C}$ film (Fig. 2a). This is indicative of a smaller 


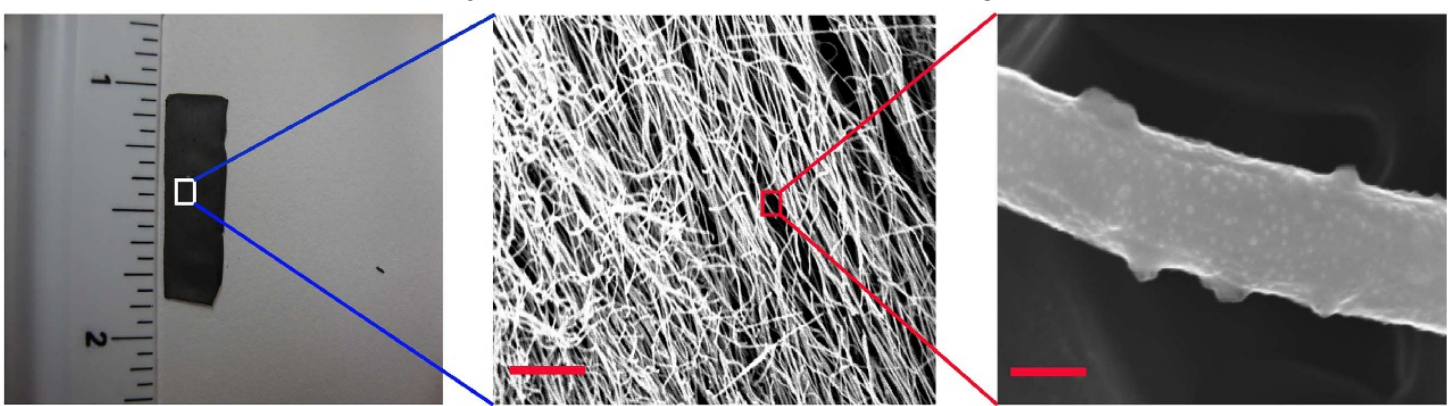

d

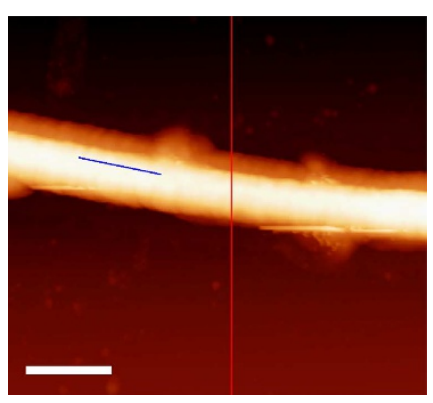

g

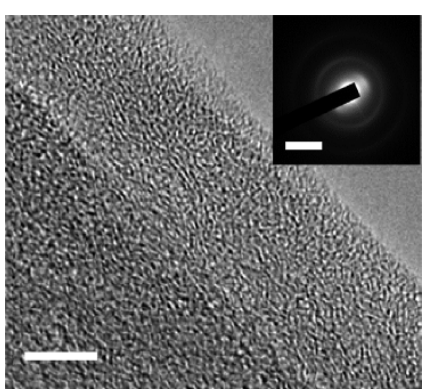

e

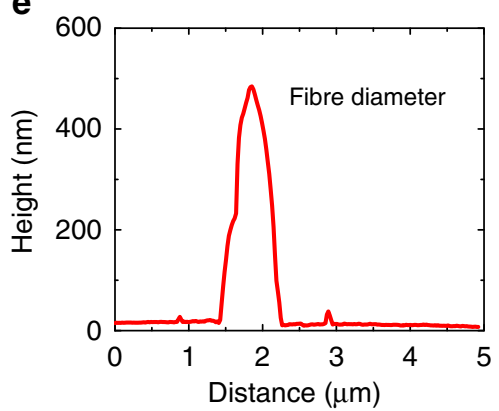

h

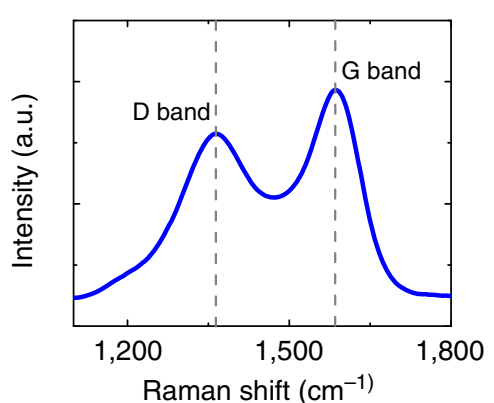

f

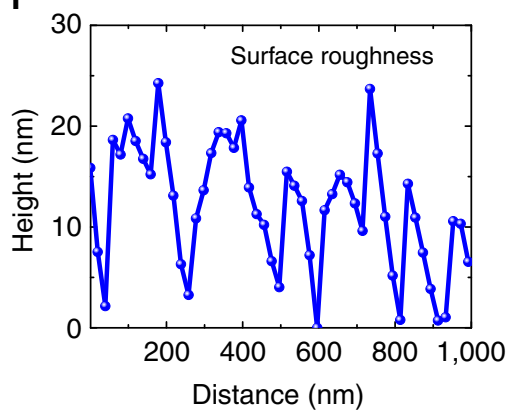

i

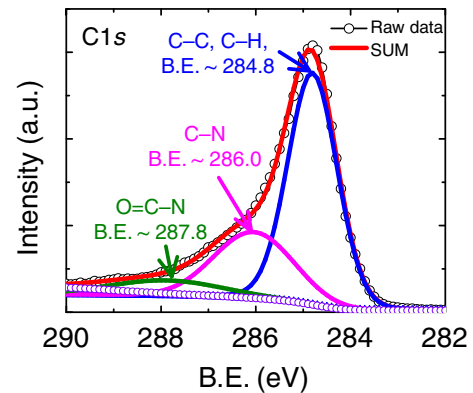

Figure 1 | Structural and elemental analysis of CNFs. (a) Digital image of CNF mat used as catalyst, (b) SEM image of the CNF mat displaying entangled fibres (scale bar, $5 \mu \mathrm{m}$ ), (c) high-resolution SEM image on individual fibres (scale bar, $200 \mathrm{~nm}$ ). Random corrugations are visible at the fibre surface. (d) Atomic force microscopic image (height profile) of fibre (scale bar, $500 \mathrm{~nm}$ ) and corresponding cross-sectional (e) and axial (f) height distributions, respectively. The fibre diameter is $\sim 500 \mathrm{~nm}$ and the height of nanocorrugations at the surface varies between 10 and $25 \mathrm{~nm}$. (g) High-resolution TEM image (scale bar, $5 \mathrm{~nm}$ ) of the edge of the fibre surface and selected area electron diffraction pattern (scale bar, $10 \mathrm{~cm}$ ) displaying disordered arrangement of crystals. (h) Two important bands in the Raman spectra (that is, D band and G band) appear at 1,363 and 1,586 cm ${ }^{-1}$. (i) Deconvoluted C1s XPS peak confirms the presence of graphitic carbon and nitrogen atoms in CNFs.

overpotential for $\mathrm{CO}_{2}$ reduction in CNFs compared with $\mathrm{C}$ film. These results also show that the overpotential in the case of CNFs is similar to that of silver $(\mathrm{Ag})$ catalyst $(0.17 \mathrm{~V})^{4}$, the smallest overpotential reported so far. Next, the current density at the highest $\mathrm{CO}_{2}$ reduction potential for $\mathrm{CNFs}$ is approximately two times higher compared with the $\mathrm{C}$ film.

Figure $2 \mathrm{~b}$ represents a comparison of the $\mathrm{CO}_{2}$ reduction (into CO) ability of noble metal Ag film, and Ag nanoparticles (200, 40 and $5 \mathrm{~nm})^{10}$, C film and CNFs electrodes. Ag catalysts are known to be the best catalyst for $\mathrm{CO}_{2}$ reduction. The comparative analysis delivers remarkably interesting information. The current density for CNFs is $\sim 13$ times higher than that of the bulk Ag film, 4 times larger than the $5-\mathrm{nm}$ Ag nanoparticles ${ }^{10}$ and twice that of the $C$ film-based electrodes (present study). It should be noted that the onset potential is similar but the potential at which maximum $\mathrm{CO}_{2}$ reduction occurs is significantly lower for CNFs $(-0.573 \mathrm{~V}$ ) in comparison with $\mathrm{Ag}$ (for $5 \mathrm{~nm} \mathrm{Ag}$ nanoparticles, it is $-0.75 \mathrm{~V})^{10}$. The potential difference between the maximum $\mathrm{CO}_{2}$ reduction and hydrogen evolution peak $(-1.14 \mathrm{~V}$ versus $\mathrm{SHE})$ for the CNF catalyst is larger $(0.567 \mathrm{~V})$ compared with $\mathrm{Ag}$ nanoparticles $(0.45 \mathrm{~V})^{10}$. This opens an adequate potential window for $\mathrm{H}_{2}$ evolution phenomena, whereas this is not the case using Ag nanoparticles. Utilization of Ag nanoparticles for $\mathrm{CO}_{2}$ reduction usually results in suppression of $\mathrm{H}_{2}$ evolution, which is mainly due to the lower potential window between the maximum $\mathrm{CO}_{2}$ reduction and $\mathrm{H}_{2}$ evolution peaks. Thus, based on the applied potential, CNFs can be used as a multifunctional catalyst system for both $\mathrm{CO}$ formation $(-0.573 \mathrm{~V}$ versus SHE) and $\mathrm{H}_{2}$ evolution $(-1.14 \mathrm{~V}$ versus $\mathrm{SHE})$ and a mixture of $\mathrm{CO}$ and $\mathrm{H}_{2}$ within the applied potential range from -0.573 to $-1.14 \mathrm{~V}$ versus SHE. This could be an efficient way of producing syngas ( $\mathrm{CO}$ and $\mathrm{H}_{2}$ mixture), which can be used directly as fuel (in gas turbines, steam power plants and so on) or as an intermediate for synthesis of higher-energy density fuels (for example, diesel, naphtha and so on). Such features were absent in Ag catalysts.

In addition, the electrochemical reduction of $\mathrm{CO}_{2}$ with respect to proton concentration by varying water mole fraction in electrolyte was also investigated (Fig. 2c). The notable advanced reduction current density (approximately five times higher) was 
a

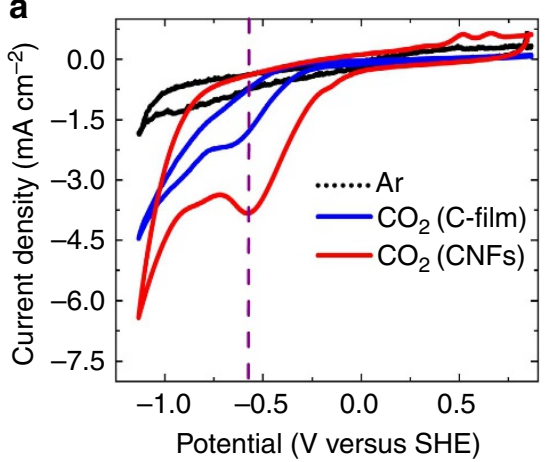

C

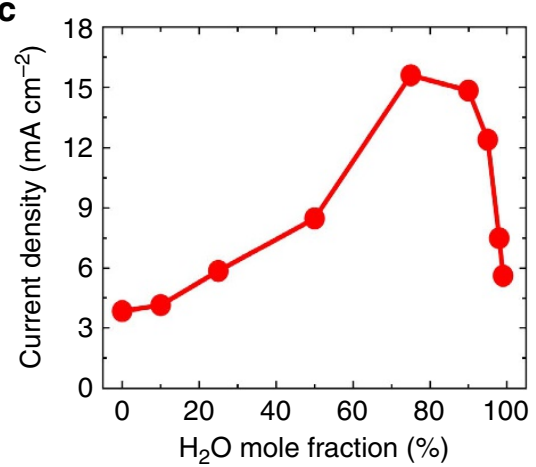

b

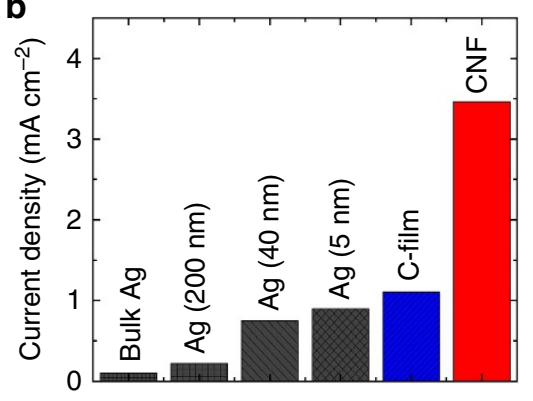

d

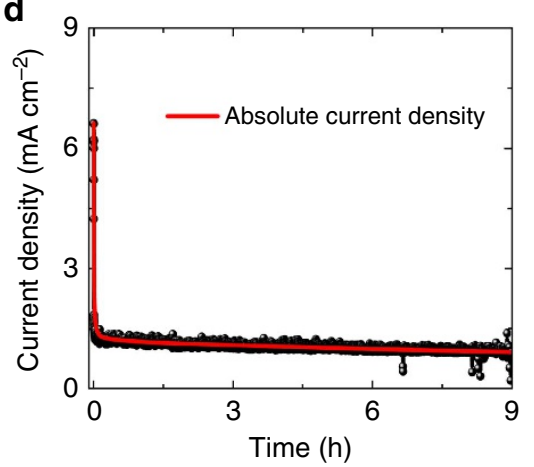

Figure 2 | Catalytic performance of $\mathbf{C N F s}$ for $\mathbf{C O}_{2}$ reduction. (a) $\mathrm{CVs}$ for $\mathrm{CO}_{2}$ reduction in the Ar-saturated (black curve) and $\mathrm{CO}_{2}$-saturated pure $\mathrm{EMIM}_{\mathrm{BF}}$ on carbon film electrode (blue curve) and CNFs electrode (red curve), respectively. The scan rate was $10 \mathrm{mVs}^{-1}$ for both experiments. The vertical pink dashed line represents the potential at which highest $\mathrm{CO}_{2}$ reduction in the case of $\mathrm{CNF}$ electrode occurs. (b) Absolute current density for $\mathrm{CO}_{2}$ reduction at different (bulk $\mathrm{Ag}, \mathrm{Ag}$ nanoparticles and $\mathrm{CNF}$ ) electrodes in pure EMIM-BF 4 electrolyte. CNFs show the highest current density and it is one order of magnitude higher compared with bulk $\mathrm{Ag}$ electrode. (c) Current density (at the highest $\mathrm{CO}_{2}$ reduction potential) for $\mathrm{CNFs}_{\mathrm{N}}$ catalyst

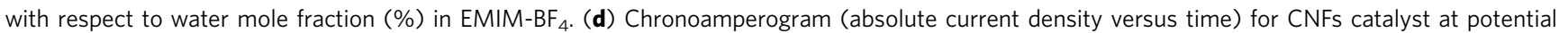
$0.573 \mathrm{~V}$ versus SHE in pure EMIM-BF 4 shows the stability of catalyst for the tested duration $(9 \mathrm{~h})$. The scan rate was $10 \mathrm{mVs}{ }^{-1}$. The current densities were obtained based on geometrical surface area.

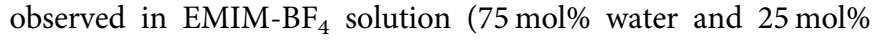
EMIM-BF 4 ) compared with the pure EMIM-BF 4 . Similar results for $\mathrm{Ag}$ nanoparticles have also been reported in our previous work $^{27}$. The addition of water molecules to EMIM-BF 4 instantly influences the $\mathrm{pH}$ (Supplementary Table S1) and viscosity of EMIM- $\mathrm{BF}_{4}$. The decrease in the $\mathrm{pH}$ of the electrolyte solution is caused by the formation of hydroxyl ions (such as $\left[\mathrm{BF}_{3} \mathrm{OH}\right]^{-}$, $\left[\mathrm{BF}_{2}(\mathrm{OH})_{2}\right]^{-}$and $\left.\left[\mathrm{BF}(\mathrm{OH})_{3}\right]^{-}\right)$because of hydrolysis of EMIM$\mathrm{BF}_{4}$. This leads to a greater proton availability, which enhances the rate of $\mathrm{CO}_{2}$ reduction.

Moreover, the stability of the catalyst is also one of the major concerns for the development of $\mathrm{CO}_{2}$ reduction system. Thus, we tested the long-term performance of CNFs catalyst by chronoamperometry (CA) at $-0.573 \mathrm{~V}$ versus SHE. The current-time CA curve was plotted for the tested duration of $9 \mathrm{~h}$ as shown in Fig. 2d. The lower magnitude of tangent $\left(\sim 1.89^{\circ}\right.$, calculated for the curve between 1 and $9 \mathrm{~h}$ ) proves that the decay in the steadystate current density (in CA curve) is negligible, indicating a higher stability similar to Ag nanoparticles (Supplementary Fig. S7) and demonstrates the renewable characteristics of our catalyst system. It is worth to mention that the initial decrease in the current density (in Fig. 2d) is due to the mass transport limitation to the electrodes in the absence of stirring or mixing the electrolyte. Once the reactants immediately adjacent to the electrode are consumed, the $\mathrm{CO}_{2}$ reduction reaction rate is governed by the diffusion of reactant from the bulk to the electrode surface ${ }^{28}$. Thus, a 'steady-state current density' is preserved afterwards. Further, a careful evaluation of CV (Fig. 2a) and CA (Fig. 2d) results illustrates that the current density for $\mathrm{CO}_{2}$ reduction in $\mathrm{CV}$ experiments is more than twice that of the values achieved in CA experiments. It should be noted that the total current measured in CV mode is a combination of Faradaic and charging currents. The charging current arises because of the fluctuation in the double layer capacitance as the applied voltage is changed in $\mathrm{CV}$. However, in a CA experiment (constant potential), the charging current is short lived and decays to a negligible value after several seconds. Thus, the superior current density observed in $\mathrm{CV}$ experiments can be attributed to the presence of extra current (due to interface charging) while it is absent in CA experiments ${ }^{28}$.

In the next experiment, we measured the work function of CNFs mats and bulk Ag by ultraviolet photoelectron spectroscopy (UPS). Our results indicate that the work function of CNFs $(\sim 4.52 \mathrm{eV})$ is higher than the work function of bulk $\mathrm{Ag}(4.33 \mathrm{eV})$. Basically, the binding energies to the catalyst surface are proportional to the average of the electron affinity (work function) of the surface and the electron affinity of the adsorbents. Higher work functions lead to higher binding energies because the energy gained by sharing electrons with the surface is large. Thus, we attribute the high $\mathrm{CO}_{2}$ reduction performance of CNFs to its unique structural features such as fibrillar morphology, rough surface (due to the fractal-like corrugations on the surface), high number of active catalytic sites and high B.E. of key intermediates to the CNF surface ${ }^{10}$.

There are a number of recent reports that explain the low overpotential $\mathrm{CO}_{2}$ reduction mechanism under various 
conditions for example, homogenous ${ }^{11,12}$ or heterogeneous ${ }^{4,10,26}$ system. In particular, Cole et. al ${ }^{12}$ suggested that $\mathrm{CO}_{2}$ conversion in pyridine (liquid) occurs via intermediate pyridinium radical (at negative potential) that further reacts with $\mathrm{CO}_{2}$ homogeneously to yield products. Rosen et. al $l^{4,26}$ proposed that $\mathrm{CO}_{2}$ reduction executed in EMIM-BF 4 (electrolyte) occurs through the formation of [EMIM- $-\mathrm{CO}_{2}$ ] intermediate followed by metal catalyst-induced reduction. However, neither of the mechanisms could fully elucidate the $\mathrm{CO}_{2}$ reduction in the present study because of the heteroatomic nature of our catalyst. The main question, in fact, is how CNFs and intermediate components $\left[\mathrm{EMIM}-\mathrm{CO}_{2}\right.$ ] take part in $\mathrm{CO}_{2}$ reduction reaction ${ }^{26}$.

\section{Discussion}

There are two electrochemically active species in CNFs catalysts: (i) pyridinic nitrogen and (ii) positively charged carbon atoms. As to counterbalance the high negative charge density on pyridinic $\mathrm{N}$, the adjacent carbon atom carries a substantially higher positive charge density and is an oxidized carbon. If the intriguing $\mathrm{CO}_{2}$ conversion observed for CNFs is associated to the nitrogen functional groups, the nature of nitrogen atoms should be significantly changed (after the experiment). To examine this, we measured the change in $\mathrm{N}$ atom configuration of CNFs catalyst by recording the high-resolution N1s spectra before and after $9 \mathrm{~h}$ electrochemical reaction (Fig. 3). Figure 3a elucidates the existence of three main nitrogen species that is, pyridinic (B.E. $\sim 398.5 \mathrm{eV}$ ), quaternary (B.E. $\sim 401.1 \mathrm{eV})$ and nitrogen oxides (B.E. $\sim 402.2 \mathrm{eV}$ ) in $\mathrm{CNFs}^{29,30}$. The quantitative analyses demonstrate that the fraction of the various nitrogen species is approximately: $\quad 25.8 \%$-pyridinic, $\quad 36.7 \%$-quaternary and $37.5 \%-\mathrm{N}$-oxide. Interestingly, the $\mathrm{N} 1 \mathrm{~s}$ spectrum (Fig. 3b) acquired for CNFs after $9 \mathrm{~h}$ reaction exhibits an additional strong $\mathrm{N}$ peak (pyridonic nitrogen) detected at B.E. $(\sim 400.1 \mathrm{eV})$. Our analysis shows that the area of $\mathrm{N}$-oxide peak considerably decreases (37.5-10\%) indicating that $\mathrm{N}$-oxides are converted to pyridonic nitrogen (peak area changes from 0 to $37 \%$ ). However, the electrochemical activity of the CNF catalysts remains unchanged, as proven by CA experiments (Fig. 2d), suggesting that $\mathrm{N}$-oxides are not taking part in the $\mathrm{CO}_{2}$ conversion process. Remarkably, the intensity of the pyridinic (highly active) and quaternary nitrogen (less active) peaks remain approximately constant after the $9 \mathrm{~h}$ experiments. If pyridinic nitrogen would directly participate in the reaction, there could be two possible mechanisms. First, pyridinic nitrogen could be permanently protonated, generating quaternary nitrogen ${ }^{12}$ resulting in an enhancement in the peak area of quaternary nitrogen, as the B.E. for protonated nitrogen is similar to quaternary (that is, $\sim 401.3 \mathrm{eV})^{30}$. Alternatively, instead of becoming permanently protonated, pyridinic nitrogens could weakly bind to the $\mathrm{CO}_{2}$ species in a similar way as in $\mathrm{CO}_{2}$ reduction by pyridine (homogenous catalyst), resulting in conversion of pyridinic nitrogen into pyridonic species ${ }^{12}$. In both scenarios, a reduction in the peak area of the pyridinic nitrogen peak (after experiments) would have been observed but it remains unchanged $(\sim 25.8 \%)$ evidencing that such reactions do not take place in our system. These results suggest that direct participation of the nitrogen group in the $\mathrm{CO}_{2}$ reduction violates the observed renewability of the $\mathrm{CO}_{2}$ reduction cycle. Thus, N1s spectra analyses force us to consider that the electrochemical reduction of $\mathrm{CO}_{2}$ is led by the positively charged carbon atoms. This is further confirmed as nitrogen-free carbon atom catalysts (for example, graphite) exhibit negligible $\mathrm{CO}_{2}$ reduction current density (Supplementary Fig. S8). The theoretical ${ }^{18,19}$ and experimental ${ }^{15,31,32}$ studies have also proven that because of high atomic charge and spin density, oxidized carbon atoms can work as effective catalysts for reduction reaction. Initially, the naturally oxidized carbon atoms can be reduced by redox cycling process. At the second step, intermediate complex [EMIM- $\left.-\mathrm{CO}_{2}\right]$ will adsorb on reduced carbon atoms and re-oxidize them to their initial state and yield $\mathrm{CO}$ as a product
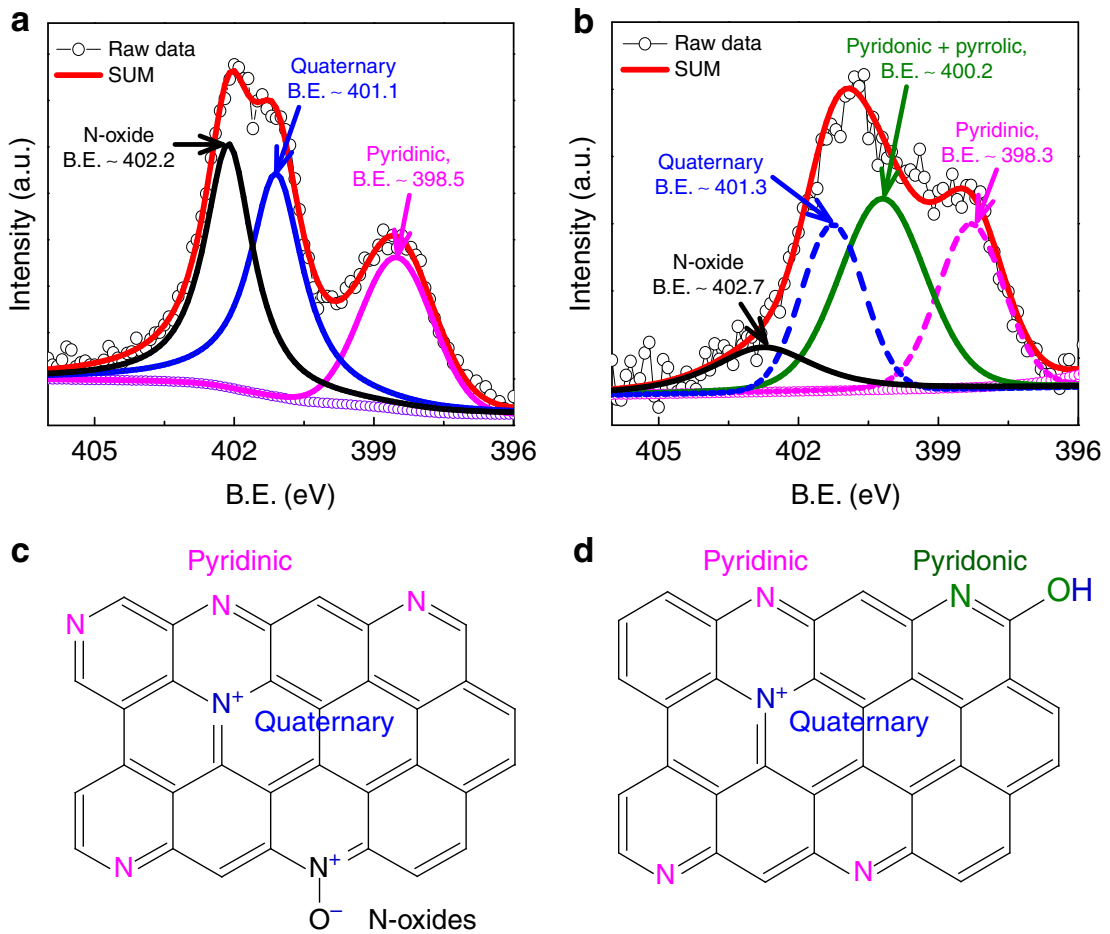

Figure 3 I Evolution of nitrogen atomic nature in CNFs by XPS. (a) Deconvoluted N1s spectra for CNFs before and (b) after electrochemical experiments. In used catalysts CNFs N1s spectra, N-oxide type of nitrogen content reduced radically and new peak (green solid line) at 400.2 eV (pyridonic N) appears. (c,d) The corresponding atomic structure on the basis of XPS analysis. 


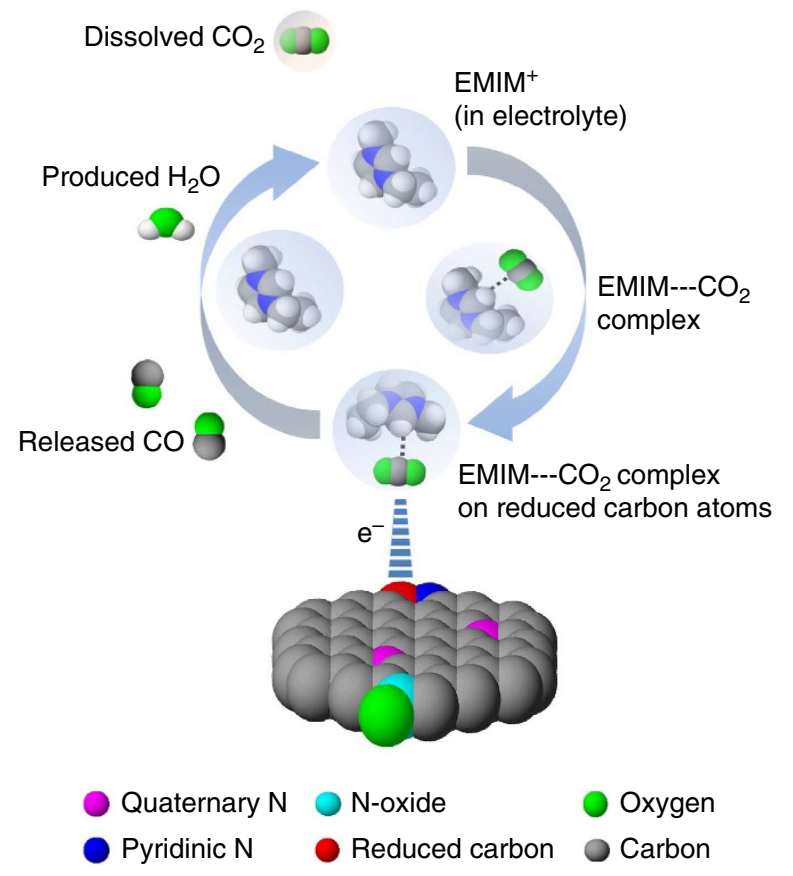

Figure $4 \mid \mathrm{CO}_{2}$ reduction mechanism schematic diagram. The $\mathrm{CO}_{2}$ reduction reaction takes place in three steps: (1) an intermediate (EMIM$\mathrm{CO}_{2}$ complex) formation, (2) adsorption of $\mathrm{EMIM}-\mathrm{CO}_{2}$ complex on the reduced carbon atoms and (3) $\mathrm{CO}$ formation.

(Fig. 4). The cyclic reduction and oxidation process keep the availability of catalytic active carbon atoms for new intermediate complex [EMIM- $\mathrm{CO}_{2}$ ] adsorption. As a result of the incessant renewable process of active carbon atoms, the $\mathrm{CO}_{2}$ reduction current density remains almost constant even after $9 \mathrm{~h}$ (Fig. 2d). This is consistent with a previous report on ORR ${ }^{15}$.

In conclusion, we have uncovered that CNFs, as a metal-free and non-precious catalyst, exhibits exceptionally high current density ( $\sim 13$ times higher than bulk $\mathrm{Ag}$ ) for a selective conversion of $\mathrm{CO}_{2}$ to $\mathrm{CO}$ (at $-0.573 \mathrm{~V}$ versus SHE). CNFs can also be potentially used to produce $\mathrm{H}_{2}$ or a mixture of $\mathrm{CO}$ and $\mathrm{H}_{2}$ (syngas) via tuning the applied potential in the range of -0.573 to $-1.14 \mathrm{~V}$ versus SHE. The catalytic proficiency of CNFs is attributed to the highly positive charges on carbon atoms due to the existence of the electronegative nitrogen atoms within the carbon lattice. Further, the extremely high $\mathrm{CO}_{2}$ reduction performance of CNFs catalyst is attributed to the nanofibrillar structure coupled with fractal-like corrugations of the individual fibres, and thus to large extent of active sites. It has also been shown that CNFs exhibits high work function that leads to higher binding energies between the $\mathrm{CO}_{2}$ intermediate complex and the CNF surface. We propose that non-precious CNFs can successfully replace the expensive noble metal electrodes with the promise of higher performance in the $\mathrm{CO}_{2}$ conversion. The study also opens up a new avenue for the development of carbon-based heteroatomic catalysts, which can be engineered either by inserting single electronegative components (N, O, S and so on) or a combination of these heteroatoms, for improved $\mathrm{CO}_{2}$ conversion efficiency.

\section{Methods}

Preparation of CNFs. The CNFs were synthesized by electrospinning of polyacrylnitrile (PAN) polymer solution followed by carbonizing the resulted electrospun mat at $1,050^{\circ} \mathrm{C}$ in Ar environment ${ }^{33,34}$. In brief, a 12 -wt\% PAN solution was prepared in $N, N$-dimethylformamide. The nanofibre mat was obtained by electrospinning the PAN solution. During this process, the electric field strength was kept as $\sim 1-2 \mathrm{kV} \mathrm{cm}^{-1}$. For carbonization, the electrospun nanofibre mat was heated to $3500^{\circ} \mathrm{C}$ in open air under the heating rate of $7.5^{\circ} \mathrm{C} \mathrm{min}^{-1}$. After it was left at $350^{\circ} \mathrm{C}$ for $3 \mathrm{~h}$, the temperature was raised to $1,050^{\circ} \mathrm{C}$ at a heating rate of $5^{\circ} \mathrm{C} \min ^{-1}$ in Ar atmosphere. The sample was kept at $1,050^{\circ} \mathrm{C}$ for $1 \mathrm{~h}$ and then cooled to room temperature in $\mathrm{Ar}$ atmosphere. The nanoscaled fractal-like surfaces of the individual nanofibres form because the carbonization chamber still contains residual oxygen, which partially oxidizes the carbonized surface, whereas the $\mathrm{Ar}$ flow blows off loosely connected flakes resulting from this partial surface oxidation. Similar CNFs were used as electrodes in microbial fuel cells and Li-ion batteries, where an enhanced performance was revealed for other electrochemical reactions ${ }^{34-36}$. The C film was also synthesized by carbonizing the film produced from the same solution used to form CNFs followed by similar carbonizing methodology. For $\mathrm{C}$ film, the normalized weight with respect to the geometrical surface area was $\sim 16 \mathrm{mg} \mathrm{cm}^{-2}$, whereas it was $\sim 2.5 \mathrm{mg} \mathrm{cm}^{-2}$ for CNFs sample. The obtained samples were cut in rectangles $1 \times 0.5 \mathrm{~cm}^{2}$ to be used as electrodes for further electrochemical $\left(\mathrm{CO}_{2}\right.$ reduction) experiments.

Morphological characterization. At first, SEM was performed to characterize the morphology of the C film and CNFs. The instrument used for characterization is integrated in a Raith e-LiNE plus ultra-high-resolution electron beam lithography system. During imaging, the samples were kept at a distance of $10 \mathrm{~mm}$ from the electron source and the voltage was kept at $10 \mathrm{kV}$. No particular types of preparation were implemented before imaging. Supplementary Fig. S2 displays the featureless morphology (as expected) of C film compared with CNFs (see Fig. 1b) synthesized by carbonization of PAN (as precursor).

The high-resolution transmission electron microscopy image and selected area electron diffraction pattern of CNFs were acquired by using a JEM-3010 (a 300-kV TEM) with a LaB6 electron source. The TEM was operated at $60 \mathrm{kV}$ accelerating voltage. The samples were prepared by dispersing CNFs fraction in ethanol and the solution was deposited on the holey carbon grid by dripping a dispersion drop. It is emphasized that all the TEM images were captured over the holes in the holey carbon grid, which allowed us to avoid the contrast associated with the carbon film used for support. Images were acquired using a Gatan digital imaging system through Digital Micrograph software.

CNFs were also visualized at nanoscale with a Nanoscope IIIa atomic force measurement(atomic force microscopic) digital instrument. First, samples were prepared by dispersing CNFs in methanol followed by drop casting on cleaned $\mathrm{Si}$ substrates. The samples were scanned in tapping mode under ambient conditions with a ratio of set point amplitude to the free amplitude of 0.9. Silicon probes Tap190G tips (budget sensor) with typical resonance frequency $190 \mathrm{kHz}$ and radius between 5 and $10 \mathrm{~nm}$ were used. Diameters and surface roughness of CNFs were analysed using the section analysis software of the microscope (WSxM 5.0-from Nanotech). Numerous individual CNFs were characterized. The diameter of CNFs varies in the ranges $400-550 \mathrm{~nm}$.

Raman spectroscopy. Raman spectroscopy (Renishaw Raman 2000) was used to detect the graphitic carbon structure and presence of defects for both CNFs and C film. The spectrum was obtained by exposing small pieces of the samples of CNFs and C film (without any particular treatment) to a 514-nm laser beam (Ar laser, power $10 \mathrm{~mW}$ and spot size $10 \mu \mathrm{m}$ ).

X-ray photoelectron spectroscopy. To execute elemental analysis, XPS experiments were performed using the Kratos Axis-165 instrument. The base pressure of the system was less than $2 \times 10^{-10}$ Torr, whereas the pressure during analysis never exceeds $2 \times 10^{-9}$ Torr. Samples (before and after electrochemical experiments) were irradiated by a monochromatic $\mathrm{Al}-\mathrm{K}_{\alpha} \mathrm{X}$-ray source $(15 \mathrm{kV}, 10 \mathrm{~mA})$. The angle of monochromatic light was fixed at $60^{\circ}$ with respect to the sample surface. Photoelectrons were detected by eight channeltrons of the concentric hemispherical analyser over an area of $700 \times 300 \mu \mathrm{m}^{2}$, with a spectrometer take-off angle of zero (with respect to the sample surface normal). The detection was achieved using the constant analyser energy mode. Survey scans were acquired with a pass energy of $160 \mathrm{eV}, 1.0 \mathrm{eV}$ step-size and $100 \mathrm{~ms}$ dwell time; whereas narrow scans were acquired with a pass energy of $20 \mathrm{eV}, 0.1 \mathrm{eV}$ step-size and $200 \mathrm{~ms}$. All scans were performed with the charge-neutralization system running. Charge referencing was done with the adventitious carbon peak position of $284.8 \mathrm{eV}$. Peak fittings were accomplished using the XPSPEAK version 4.1 program. Baselines were fitted using Shirley-typed backgrounds. All XPS fitting data could be described by a mixture of Gaussian-Lorentzian distributions with typical ratios of $70 \%$ Gaussian or higher.

Electrochemical experiments. To monitor the $\mathrm{CO}_{2}$ reduction, the following procedure was adapted. Ultra high pure (UHP) $\mathrm{Ar}$ (purchased from AirGas) was initially sparged at $1 \mathrm{sl} \mathrm{min}^{-1}$ flow rate and 10 p.s.i. pressure for $2 \mathrm{~h}$ until a saturated condition was reached to ensure that all the oxygen was removed from the ionic liquid. CV curve was then taken using a Voltalab PGZ100 potentiostat (purchased from Radiometer Analytical SAS) calibrated with a RCB200 resistor capacitor box. The potentiostat was connected to a personal computer using Volta Master (version 4) software. The obtained curve was used as a reference curve. An aerobic $\mathrm{CO}_{2}$ (AirGas) was then bubbled in the solution at the same flow rate and 
pressure for $30 \mathrm{~min}$. The $\mathrm{CV}$ curve was then acquired. For better mixing, slight magnetic stirring was applied throughout the entire process. CNFs or C film with $1 \times 0.5 \mathrm{~cm}^{2}$ surface area were used as cathode. The connection between the cathode and the potentiostat was performed using an aluminium crocodile clamp. A $25 \times 8-\mathrm{mm}^{2}$ piece of platinum, gauze, 52 mesh with $99.9 \%$ metal basis (purchased from Sigma-Aldrich) was used as a counter electrode. The reference electrode was a silver wire $(0.5 \mathrm{~mm}$ diameter, annealed, $99.9 \%$ metal basis $)$ purchased from Alfa-Aesar. A solution of ionic liquid EMIM-BF ${ }_{4}$ was used as electrolyte (purchased from Sigma-Aldrich). All chemicals were used as purchased without any further chemical treatment. Reference and counter electrodes were washed with acetone and isopropanol several times, rinsed with deionized (DI) water and dried with dry nitrogen (AirGas) before use. The schematic diagram of three-neck electrochemical cell is shown in Supplementary Fig. S9. The long-term stability of CNFs catalyst was also studied by performing CA experiment at $-0.573 \mathrm{~V}$ versus SHE in similar electrochemical cell. Additional CV experiments were performed to scan the $\mathrm{CO}_{2}$ reduction ability of nitrogen-free carbon atom catalysts that is, graphite (see below).

Nitrogen-free carbon catalyst performance. Graphite chemical structure (hexagonal $s p^{2}$ carbon rings) ideally represents the atomic structure of the highly ordered crystallized CNFs. Thus, to do the experiments with nitrogen-free carbon materials, we have chosen graphite as a catalyst. Graphite with $>99.9$ purity was purchased from the Electron Microscopy Science Company. The cyclic voltammetric (CV) experiments were performed under similar condition (for example, pure ionic liquid-EMIM-BF 4 ) as for CNFs. The typical CV curves obtained in UHP argon and $\mathrm{CO}_{2}$-saturated electrolyte are presented in Supplementary Fig. S8. As expected, both $\mathrm{CV}$ curves are fully overlapped to each other, indicating that $\mathrm{CO}_{2}$ reduction reaction does not take place on the graphite catalyst surface. The observed current density at higher potential can be attributed to the well-studied graphite activity towards hydrogen evolution reaction as reported in previous reports ${ }^{37,38}$. These results clearly verify that $\mathrm{CO}_{2}$ reduction on the nitrogen-free catalyst (graphite) is negligible under the similar experimental conditions.

Long-term chronoamperometric study for Ag nanoparticles. We also perform the long-term CA experiments for the silver nanoparticles (Ag NPs) to compare the performance with CNFs. The catalyst ink was prepared by mixing $40 \mathrm{mg}$ of Ag NPs purchased from the Aldrich ( $\sim 40 \mathrm{~nm}$ diameter) with $2.4 \mathrm{ml}$ of DI water and $2.4 \mathrm{ml}$ of isopropyl alcohol. To prepare the homogenous dispersion, this mixture was sonicated for $2 \mathrm{~min}$. The electrode was prepared by painting Ag NPs ink onto a piece of Sigracetgraphite layer with $1 \mathrm{~cm}^{2}$ surface area. The back side of the substrate was fully covered with Hysol 9460 epoxy adhesive. Before measurements, the samples were immersed in $0.1 \mathrm{M}$ sulphuric acid overnight and rinsed with DI water to remove impurities. Electrochemical experiments for Ag NPs were performed in the same condition as for the CNFs samples. As shown in Supplementary Fig. S7, the magnitude of tangent $\sim 1.84^{\circ}$ calculated for the curve between 1 and $9 \mathrm{~h}$ is very close to the stability decay slope calculated for CNFs catalysts $\left(\sim 1.89^{\circ}\right)$.

Gas chromatography. $\mathrm{CO}$ formation due to $\mathrm{CO}_{2}$ reduction was verified by GC in SRI 8610C GC system. The SRI thermal conductivity detector (TCD) consisting of four filaments was set in high current mode. UHP helium (purchased from AirGas) at $10 \mathrm{ml} \mathrm{min}-1$ flow rate was used as a carrier gas. After the TCD detector oven reached $100^{\circ} \mathrm{C}$ and stabilized, $2 \mathrm{ml}$ of gas sample was injected to the GC at minute 0.18 and a clear CO peak appeared at minute 3.83 .

Ultraviolet photoelectron spectroscopy. Surface work function measurements were conducted using UPS. The UPS data were acquired with a Physical Electronics PHI 5400 photoelectron spectrometer using He I $(21.2 \mathrm{eV})$ ultraviolet radiation and a pass energy of $8.95 \mathrm{eV}$. To separate the signal arising from secondary electron emission from the detector from the secondary electron emission from the sample, a $-9-\mathrm{V}$ bias was applied to the sample using a battery.

\section{References}

1. Davis, S. J., Caldeira, K. \& Matthews, H. D. Future $\mathrm{CO}_{2}$ emissions and climate change from existing energy infrastructure. Science 329, 1330-1333 (2010).

2. Benson, E. E., Kubiak, C. P., Sathrum, A. J. \& Smieja, J. M. Electrocatalytic and homogeneous approaches to conversion of $\mathrm{CO}_{2}$ to liquid fuels. Chem. Soc. Rev. 38, 89-99 (2009).

3. Whipple, D. T. \& Kenis, P. J. A. Prospects of $\mathrm{CO}_{2}$ utilization via direct heterogeneous electrochemical reduction. J. Phys. Chem. Lett. 1, 3451-3458 (2010).

4. Rosen, B. A. et al. Ionic liquid-mediated selective conversion of $\mathrm{CO}_{2}$ to $\mathrm{CO}$ at low overpotentials. Science 334, 643-644 (2011).

5. Richardson, R. D., Holland, E. J. \& Carpenter, B. K. A renewable amine for photochemical reduction of $\mathrm{CO}_{2}$. Nat. Chem. 3, 301-303 (2011).

6. Hori, Y., Murata, A., Kikuchi, K. \& Suzuki, S. Electrochemical reduction of carbon dioxides to carbon monoxide at a gold electrode in aqueous potassium hydrogen carbonate. J. Chem. Soc. Chem. Commun. 10, 728-729 (1987).
7. Li, C. W. \& Kanan, M. W. $\mathrm{CO}_{2}$ reduction at low overpotential on Cu electrodes resulting from the reduction of thick $\mathrm{Cu}_{2} \mathrm{O}$ films. J. Am. Chem. Soc. 134, 7231-7234 (2012).

8. Hoshi, N., Suzuki, T. \& Hori, Y. Step density dependence of $\mathrm{CO}_{2}$ reduction rate on $\mathrm{Pt}(\mathrm{S})-[\mathrm{n}(111) \times(111)]$ single crystal electrodes. Electrochim. Acta 41, $1647-1653$ (1996).

9. Simón-Manso, E. \& Kubiak, C. P. Dinuclear nickel complexes as catalysts for electrochemical reduction of carbon dioxide. Organometallics 24, 96-102 (2005).

10. Salehi-Khojin, A. et al. Nanoparticle silver catalysts that show enhanced activity for carbon dioxide electrolysis. J. Phys. Chem. C 117, 1627-1632 (2013).

11. Seshadri, G., Lin, C. \& Bocarsly, A. B. A new homogeneous electrocatalyst for the reduction of carbon dioxide to methanol at low overpotential. J. Electroanal. Chem. 372, 145-150 (1994).

12. Cole, E. B. et al. Using a one-electron shuttle for the multielectron reduction of $\mathrm{CO}_{2}$ to methanol: kinetic, mechanistic, and structural insights. J. Am. Chem. Soc. 132, 11539-11551 (2010).

13. Riduan, S. N., Zhang, Y. \& Ying, J. Y. Conversion of carbon dioxide into methanol with silanes over $\mathrm{N}$-heterocyclic carbene catalysts. Angew. Chem. Int. Ed. 48, 3322-3325 (2009).

14. Wang, W., Wang, S., Ma, X. \& Gong, J. Recent advances in catalytic hydrogenation of carbon dioxide. Chem. Soc. Rev. 40, 3703-3727 (2011).

15. Gong, K., Du, F., Xia, Z., Durstock, M. \& Dai, L. Nitrogen-doped carbon nanotube arrays with high electrocatalytic activity for oxygen reduction. Science 323, 760-764 (2009).

16. Geng, D. et al. High oxygen-reduction activity and durability of nitrogen-doped graphene. Energy Environ. Sci. 4, 760-764 (2011).

17. Yang, S. et al. Efficient synthesis of heteroatom (N or S)-doped graphene based on ultrathin graphene oxide-porous silica sheets for oxygen reduction reactions. Adv. Funct. Mater. 22, 3634-3640 (2012).

18. Wang, S. et al. BCN graphene as efficient metal-free electrocatalyst for the oxygen reduction reaction. Angew. Chem. Int. Ed. 51, 4209-4212 (2012).

19. Zhang, L., Niu, J., Dai, L. \& Xia, Z. Effect of microstructure of nitrogen-doped graphene on oxygen reduction activity in fuel cells. Langmuir 28, 7542-7550 (2012).

20. Smith, R. D. L. \& Pickup, P. G. Nitrogen-rich polymers for the electrocatalytic reduction of $\mathrm{CO}_{2}$. Electrochem. Commun. 12, 1749-1751 (2010).

21. Zussman, E., Yarin, A. L., Bazilevsky, A. V., Avrahami, R. \& Feldman, M. Electrospun polyacrylonitrile/poly(methyl methacrylate)-derived carbon micro-/nanotubes. Adv. Mater. 18, 348-353 (2006).

22. Blanchard, L. A., Gu, Z. \& Brennecke, J. F. High-pressure phase behavior of ionic liquid/ $\mathrm{CO}_{2}$ systems. J. Phys. Chem. B 105, 2437-2444 (2001).

23. Reich, S. \& Thomsen, C. Raman spectroscopy of graphite. Philos. T Roy. Soc. A 362, 2271-2288 (2004).

24. Wang, Y., Serrano, S. \& Santiago-Avilés, J. J. Raman characterization of carbon nanofibres prepared using electrospinning. Synthetic Met. 138, 423-427 (2003).

25. Zhong, M. et al. Electrochemically active nitrogen-enriched nanocarbons with well-defined morphology synthesized by pyrolysis of self-assembled block copolymer. J. Am. Chem. Soc. 134, 14846-14857 (2012).

26. Rosen, B. A. et al. In situ spectroscopic examination of a low overpotential pathway for carbon dioxide conversion to carbon monoxide. J. Phys. Chem. C 116, 15307-15312 (2012).

27. Rosen, B. A., Zhu, W., Kaul, G., Salehi-Khojin, A. \& Masel, R. I. Water enhancement of $\mathrm{CO}_{2}$ conversion on silver in 1-ethyl-3-methylimidazolium tetrafluoroborate. J. Electrochem. Soc. 160, H138-H141 (2012).

28. Bard, A. J. Electrochemical Methods: Fundamentals and Applications (Wiley, 2001).

29. Stańczyk, K., Dziembaj, R., Piwowarska, Z. \& Witkowski, S. Transformation of nitrogen structures in carbonization of model compounds determined by XPS Carbon N. Y. 33, 1383-1392 (1995).

30. Pels, J. R., Kapteijn, F., Moulijn, J. A., Zhu, Q. \& Thomas, K. M. Evolution of nitrogen functionalities in carbonaceous materials during pyrolysis. Carbon $N$. Y. 33, 1641-1653 (1995).

31. Zhang, L. \& Xia, Z. Mechanisms of oxygen reduction reaction on nitrogendoped graphene for fuel cells. J. Phys. Chem. C 115, 11170-11176 (2011).

32. Winther-Jensen, B., Winther-Jensen, O., Forsyth, M. \& MacFarlane, D. R. High rates of oxygen reduction over a vapor phase-polymerized PEDOT electrode. Science 321, 671-674 (2008).

33. Reneker, D. H., Yarin, A. L., Zussman, E. \& Xu, H. Electrospinning of nanofibres from polymer solutions and melts. Adv. Appl. Mech. 41, 43-195 (2007).

34. Chen, S. et al. Electrospun and solution blown carbon nanofibre nonwovens for application as electrodes in microbial fuel cells. Energy Environ. Sci. 4, 1417-1421 (2011).

35. Dimesso, L., Spanheimer, C., Jaegermann, W., Zhang, Y. \& Yarin, A. L. $\mathrm{LiFePO}_{4}-3 \mathrm{D}$ carbon nanofibre composites as cathode materials for Li-ions batteries. J. Appl. Phys. 111, 064307 (2012). 
36. Dimesso, L., Spanheimer, C., Jaegermann, W., Zhang, Y. \& Yarin, A. L. $\mathrm{LiCoPO}_{4}-3 \mathrm{D}$ carbon nanofibre composites as possible cathode materials for high voltage applications. Electrochim. Acta 95, 38-42 (2013).

37. Chen, Fuyu, Liu, Jianguo, Chen, H. u. i. \& Yan, Chuanwei Study on hydrogen evolution reaction at a graphite electrode in the all-vanadium redox flow battery. Int. J. Electrochem. Sci. 7, 3750-3764 (2012).

38. Bianco, P. Electrocatalytic hydrogen-evolution at the pyrolytic graphite electrode in the presence of hydrogenase. J. Electrochem. Soc. 139, 2428-2432 (1992).

\section{Acknowledgements}

This work was supported in part by University of Illinois at Chicago. This work was carried out in part in the Frederick Seitz Materials Research Laboratory Central Facilities, University of Illinois.

\section{Author contributions}

A.S.-K, B.K., M.A. and A.L.Y. conceived the idea. B.K., M.A. and D.P. performed the electrochemical experiments and characterizations, and A.S.-K supervised their efforts
S.S.-R. synthesized the CNFs and participated in the explanation of the role of nitrogen, and characterized CNFs by TEM and selected area electron diffraction. A.L.Y. supervised S.S.-R.'s efforts and proposed to compare the results of CNFs with C films. J.A. supervised M.A.'s efforts. R.H. performed UPS measurements. B.R. took some experiments to verify the results and participate in the discussion of electrochemical results. All authors contributed to the manuscript before submission.

\section{Additional information}

Supplementary Information accompanies this paper at http://www.nature.com/ naturecommunications

Competing financial interests: The authors declare no competing financial interests.

Reprints and permission information is available online at http://npg.nature.com/ reprintsandpermissions/

How to cite this article: Kumar, B. et al. Renewable and metal-free carbon nanofibre catalysts for carbon dioxide reduction. Nat. Commun. 4:2819 doi: 10.1038/ncomms3819 (2013) 